\title{
Long-term treatment with ruxolitinib for patients with myelofibrosis: 5-year update from the randomized, double-blind, placebo-controlled, phase 3 COMFORT-I trial
}

Srdan Verstovsek ${ }^{1 *}$, Ruben A. Mesa ${ }^{2}$, Jason Gotlib³, Vikas Gupta ${ }^{4}$, John F. DiPersio ${ }^{5}$, John V. Catalano6, Michael W. N. Deininger ${ }^{7,19}$, Carole B. Miller ${ }^{8}$, Richard T. Silver ${ }^{9}$, Moshe Talpaz ${ }^{10}$, Elliott F. Winton ${ }^{11}$, Jimmie H. Harvey Jr. ${ }^{12}$, Murat O. Arcasoy ${ }^{13}$, Elizabeth O. Hexner ${ }^{14}$, Roger M. Lyons ${ }^{15}$, Ronald Paquette ${ }^{16}$, Azra Raza ${ }^{17}$, Mark Jones ${ }^{18}$, Deanna Kornacki ${ }^{18}$, Kang Sun ${ }^{18}$, Hagop Kantarjian ${ }^{1}$ and for the COMFORT-I investigators

\begin{abstract}
Background: The randomized, double-blind, placebo-controlled, phase 3 COMFORT-I trial evaluated the JAK1/JAK2 inhibitor ruxolitinib in patients with intermediate-2/high-risk myelofibrosis. The primary and planned 3-year analyses of COMFORT-I data demonstrated that ruxolitinib—-the first myelofibrosis-approved therapy-reduced splenomegaly and prolonged overall survival versus placebo. Here, we present the final 5-year results.

Methods: Patients managed in Australia, Canada, and the USA were randomized centrally (interactive voice response system) 1:1 to oral ruxolitinib twice daily (15 or $20 \mathrm{mg}$ per baseline platelet counts) or placebo. Investigators and patients were blinded to treatment. The secondary endpoints evaluated in this analysis were durability of a $\geq 35 \%$ reduction from baseline in spleen volume (spleen response) and overall survival, evaluated in the intent-to-treat population. Safety was evaluated in patients who received study treatment.

Results: Patients were randomized (September 2009-April 2010) to ruxolitinib $(n=155)$ or placebo $(n=154)$. At termination, $27.7 \%$ of ruxolitinib-randomized patients and $25.2 \%(28 / 111)$ who crossed over from placebo were on treatment; no patients remained on placebo. Patients randomized to ruxolitinib had a median spleen response duration of 168.3 weeks and prolonged median overall survival versus placebo (ruxolitinib group, not reached; placebo group, 200 weeks; HR, 0.69; $95 \% \mathrm{Cl}, 0.50-0.96 ; P=0.025)$ despite the crossover to ruxolitinib. The ruxolitinib safety profile remained consistent with previous analyses. The most common new-onset all-grade nonhematologic adverse events starting $<12$ versus $\geq 48$ months after ruxolitinib initiation were fatigue (29.0 vs $33.3 \%$ ) and diarrhea (27.8 vs $14.6 \%$ ). New-onset grade 3 or 4 anemia and thrombocytopenia both primarily occurred within the first 6 months, with no cases after 42 months. The most common treatment-emergent adverse event-related deaths in the ruxolitinib-randomized group were sepsis (2.6\%), disease progression (1.9\%), and pneumonia (1.9\%).

(Continued on next page)
\end{abstract}

\footnotetext{
* Correspondence: sverstov@mdanderson.org

'Division of Cancer Medicine, The University of Texas MD Anderson Cancer

Center, 1515 Holcombe Blvd, Unit 418, Houston, TX 77030, USA

Full list of author information is available at the end of the article
} 
(Continued from previous page)

Conclusion: The final COMFORT-I results continue to support ruxolitinib as an effective treatment for patients with intermediate-2/high-risk MF.

Trial registration: ClinicalTrials.gov, NCT00952289

Keywords: JAK, Janus kinase, Myelofibrosis

\section{Background}

Myelofibrosis (MF) is a Philadelphia chromosomenegative myeloproliferative neoplasm [1] that is often associated with splenomegaly, anemia, and burdensome symptoms that negatively affect quality of life [2, 3]. In addition, patients with MF have shortened survival compared with age- and sex-matched members of the general population [4]. Allogeneic stem cell transplantation is the only potentially curative treatment option [5]. However, transplant-related morbidity and mortality are considerable, and many patients with MF are ineligible because of their age or comorbidities.

Many patients with MF have mutations associated with dysregulation of the Janus kinase (JAK)/signal transducer and activator of transcription pathway. The most common mutations are in JAK2 (55-65\%), CALR (15-25\%), and $M P L$ (5-15\%); a relatively small subset of patients is triple negative for mutations in all three genes (10-20\%) [6-9].

Ruxolitinib is a JAK1/JAK2 inhibitor approved by the US Food and Drug Administration for patients with intermediate- or high-risk MF, including primary MF (PMF), post-polycythemia vera MF (PPV-MF), and postessential thrombocythemia MF (PET-MF), as well as patients with PV who have had an inadequate response to or are intolerant of hydroxyurea [10]. Ruxolitinib is also approved by the European Medicines Agency for the treatment of disease-related splenomegaly or symptoms in adult patients with PMF, PPV-MF, or PET-MF and for the treatment of adult patients with PV who are resistant to or intolerant of hydroxyurea [11]. Approval for MF was based on two randomized phase 3 clinical trials in patients with intermediate-2 or high-risk PMF, PPV-MF, or PET-MF $[12,13]$. Controlled Myelofibrosis Study with Oral JAK Inhibitor Treatment (COMFORT)-I was a double-blind, placebo-controlled trial, and COMFORTII was an open-label trial comparing ruxolitinib with the best available therapy. In both trials, ruxolitinib was superior to control interventions, reducing spleen size and improving MF-related symptoms and quality-of-life (QoL) measures. Spleen volume reductions and improvements in measures of QoL at week 24 in COMFORT-I were observed regardless of MF subtype, age, International Prognostic Scoring System (IPSS) risk score, Eastern Cooperative Oncology Group (ECOG) performance status, and baseline hemoglobin level, platelet count, spleen size, and JAK2V617F mutation status [14].

Long-term follow-up analyses of the COMFORT studies have indicated durable clinical benefit and are suggestive of a survival advantage with ruxolitinib treatment [15-17]. Most nonhematologic adverse events in COMFORT-I and COMFORT-II were grade 1 or 2 , with the rate generally decreasing with long-term ruxolitinib treatment $[15,16]$. Dose-dependent cytopenias were the most common hematologic adverse events. These occurred primarily within the first 12 weeks of ruxolitinib treatment and stabilized thereafter in patients continuing therapy, with hemoglobin levels returning to nearbaseline levels $[15,16]$.

This analysis reports the final long-term efficacy and safety results of COMFORT-I after 5 years of ruxolitinib treatment.

\section{Methods}

\section{Study design and patients}

The detailed study design and protocol of the randomized, double-blind, placebo-controlled, phase 3 COMFORT-I trial have been reported previously [12]. The study was conducted in 89 sites across Australia, Canada, and the USA. Briefly, patients with intermediate- 2 or high-risk MF and splenomegaly of $>5 \mathrm{~cm}$ below the left costal margin by palpation were eligible.

The protocol was reviewed and approved by each participating site's institutional review board. All patients provided written informed consent.

\section{Randomization and masking}

Patients were randomized 1:1 to ruxolitinib or matching placebo tablets by a centralized interactive voice response system (IVRS). Study investigators and patients were blinded to the treatment. Study treatments were provided in encoded bottles, and patient study drug assignments were provided to site staff by the IVRS.

\section{Procedures}

Study treatments, administered orally twice daily, were ruxolitinib (Incyte Corporation, Wilmington, DE; dosing based on baseline platelet counts: $100-200 \times 10^{9} / \mathrm{L}$, $15 \mathrm{mg}$; $>200 \times 10^{9} / \mathrm{L}, 20 \mathrm{mg}$ ) or placebo. Dose modification was allowed for efficacy and safety. Crossover from 
placebo to ruxolitinib was permitted before week 24 for protocol-defined worsening splenomegaly. After week 24 , patients with protocol-defined worsening symptomatic spleen growth either received unblinded ruxolitinib or discontinued the study; patients with protocoldefined asymptomatic spleen growth were given the option to unblind, after which they were required to receive ruxolitinib or discontinue the study.

This final analysis occurred when all the patients reached the 5-year visit or discontinued participation. Changes from baseline or crossover baseline in spleen volume were assessed by magnetic resonance imaging or computed tomography every 12 weeks from weeks 12 to 72 and every 24 weeks thereafter. Patients who had a spleen volume measurement at baseline and each time point of interest were evaluable to determine if a $\geq 35 \%$ reduction from baseline in spleen volume was achieved; all patients who withdrew before the time point were considered nonresponders.

\section{Outcomes}

The primary endpoint was the proportion of patients who achieved spleen response $(\geq 35 \%$ reduction from baseline in spleen volume) at week 24 . Secondary endpoints reported in this analysis included duration of spleen response and overall survival (OS).

Hematologic adverse events were based on laboratory abnormalities. Because the majority of the anemia and thrombocytopenia events occurred early in the study, the incidence of new-onset or worsening grade 3 or 4 anemia or thrombocytopenia was assessed at 6-month intervals in patients originally randomized to ruxolitinib. The placebo group was included in only the first 6month interval because all the patients receiving placebo discontinued or crossed over to ruxolitinib within 3 months of the primary analysis. Nonhematologic adverse events were assessed per National Cancer Institute Common Terminology Criteria for Adverse Events [18]. The incidence of nonhematologic adverse events was assessed in yearly intervals for patients originally randomized to ruxolitinib.

\section{Statistical analysis}

Changes from baseline or crossover baseline in spleen volume were summarized with descriptive statistics. Durability of spleen response and OS were calculated with the Kaplan-Meier method in the intent-to-treat population. OS was calculated based on randomized treatment. Spleen response was considered lost at the first measurement that was no longer a $\geq 35 \%$ reduction from baseline and was also a $>25 \%$ increase from the nadir. Hazard ratio with $95 \%$ confidence interval and $P$ values were calculated with the Cox proportional hazards model and the log-rank test. A subgroup analysis of
OS was conducted in patients with intermediate- 2 or high-risk MF per IPSS criteria [19].

Safety analyses were conducted in all patients who received $\geq 1$ dose of study treatment. The incidence of new-onset or worsening grade $\geq 3$ anemia and thrombocytopenia (based on laboratory data) and of new-onset or worsening all-grade and grade $\geq 3$ nonhematologic adverse events was calculated using the lifetable method. The time to the first event censored at the date of the last laboratory evaluation was used for anemia and thrombocytopenia; the earlier discontinuation or date of data cutoff was used for nonhematologic adverse events. Per the life-table method, the incidence of each adverse event was based on the effective sample size of the time interval, which was the number of patients at risk at the beginning of the interval minus half of the censored patients during the time interval.

Statistical analyses were conducted using SAS version 9.2 (SAS Institute, Cary, NC).

The trial was overseen by a data monitoring committee and is registered at ClinicalTrials.gov (NCT00952289).

\section{Role of the funding source}

Conduct of this study and editorial assistance were funded by Incyte Corporation. Incyte Corporation employees worked with external investigators in designing the study, analyzing data, and confirming accuracy of this report. The authors had full access to all the data in the study and had final responsibility for the decision to submit.

\section{Results}

\section{Patient disposition}

Patients were recruited between September 2009 and April 2010 and randomized to ruxolitinib $(n=155)$ or placebo $(n=154$; Fig. 1$)$. All patients were included in the intent-to-treat population; three patients in the placebo group were not evaluable for safety. By the time of the 3-year analysis, all evaluable patients in the placebo group had discontinued (40/151 [26.5\%]) or crossed over to ruxolitinib (111/151 [73.5\%]) [15]. The median (range) time to crossover was 39.9 (5.0-65.3) weeks. At study termination (i.e., the 5-year data cutoff), $27.7 \%$ $(43 / 155)$ of patients originally randomized to ruxolitinib and $25.2 \%$ (28/111) of those who crossed over to ruxolitinib were receiving treatment in the study. An additional four patients in the ruxolitinib-randomized group who discontinued the study transitioned to commercial ruxolitinib.

\section{Efficacy \\ Spleen response}

Among patients originally randomized to ruxolitinib, $59.4 \%(92 / 155)$ had achieved a $\geq 35 \%$ reduction in spleen 


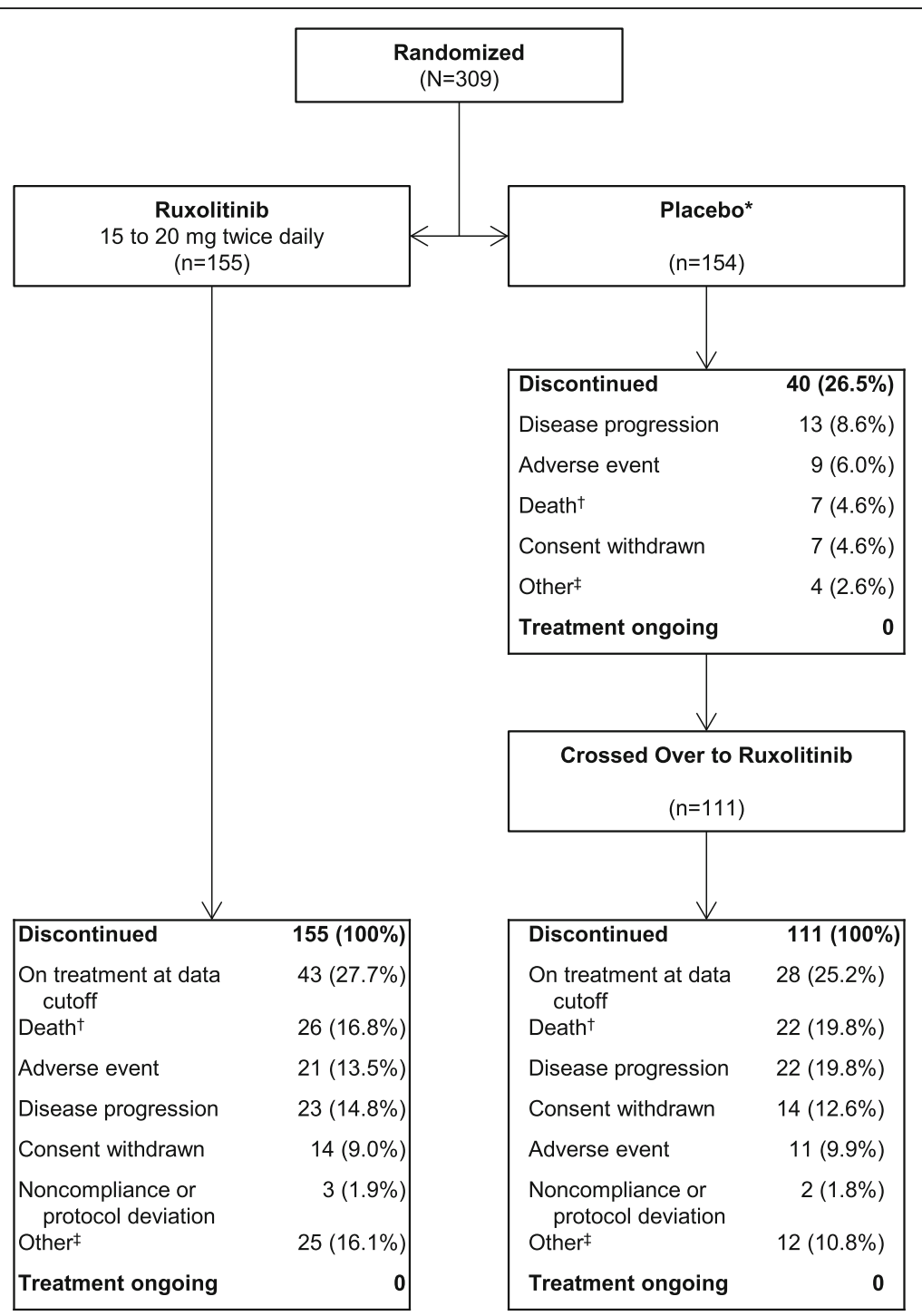

Fig. 1 Patient disposition. *Three patients in the placebo group were not evaluable for safety $(n=151)$; these patients were excluded from the calculation of the percentage of patients who discontinued. (dagger) Limited to patients whose study discontinuation dates matched their dates of death. (double dagger) Including but not limited to the following: received a different therapy, transitioned to commercial ruxolitinib, and loss of response

volume at any time during the study, with a median duration of response of 168.3 weeks (Fig. 2). The proportion of evaluable patients (i.e., those with measurements at baseline and each time point) in the ruxolitinibrandomized group who had a $\geq 35 \%$ reduction from baseline in spleen volume (including patients who had withdrawn as nonresponders) was $41.9 \%(65 / 155)$ at week $24,36.6 \%(52 / 142)$ at week $48,34.9 \%(52 / 149)$ at week $96,28.5 \%(41 / 144)$ at week 144, 22.6\% (33/146) at week 192, 20.1\% (30/149) at week 240 , and $18.5 \%$ (27/146) at week 264. Among patients continuing treatment with ruxolitinib, median percentage reductions from baseline in spleen volume were rapid and durable. In the ruxolitinib-randomized group, the median (range) percentage changes from baseline were $-33.0 \%$ $(-75.9$ to $25.1 \%)$ and $-40.8 \%(-95.9$ to $73.3 \%)$ at 24 and 240 weeks, respectively; the median (range) percentage changes from crossover baseline were $-37.3 \%$ ( -64.8 to $26.0 \%)$ and $-75.7 \%(-85.8$ to $49.1 \%)$ in the ruxolitinib crossover group (Fig. 3), although the number of evaluable patients was limited at 240 weeks $(n=9)$.

\section{Overall survival}

At the time of the final 5-year analysis, median followup time for the OS analysis was 268.4 weeks for the ruxolitinib group and 269.0 weeks for the placebo group. Patients randomized to ruxolitinib experienced prolonged OS compared with those in the placebo group. 


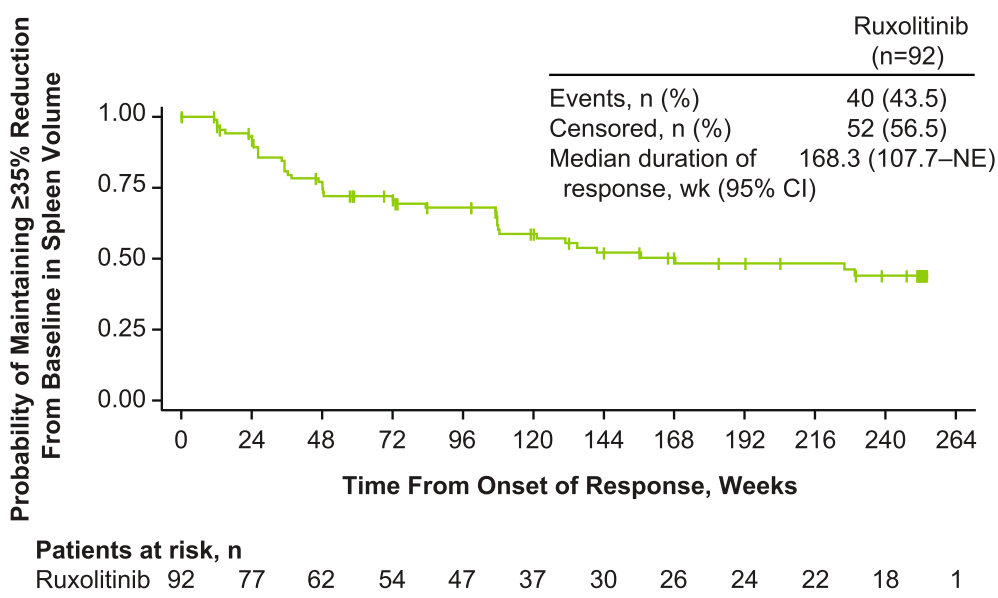

Fig. 2 Duration of $\geq 35 \%$ reduction from baseline in spleen volume. Duration of spleen response was evaluated for the 92 patients in the ruxolitinib group who achieved $\mathrm{a} \geq 35 \%$ reduction from baseline in spleen volume. NE, not evaluable

Median OS was not reached in the ruxolitinibrandomized group. Among patients randomized to placebo, median OS was 108 weeks for patients censored at crossover and 200 weeks for all patients (HR, 0.69; 95\% CI, 0.50-0.96; $P=0.025$; Fig. 4). There were a total of 69 deaths (regardless of cause) in the ruxolitinibrandomized group and 82 deaths among those randomized to placebo. In a subgroup analysis by IPSS risk status, there was a nonsignificant trend toward longer OS among patients in the ruxolitinib group compared with the placebo group for both intermediate- 2 and high-risk patients (Fig. 5).

\section{Safety}

The median (range) ruxolitinib exposure duration was 149.3 (4.3-296.0) weeks in the ruxolitinib-randomized group and $111.0(0.9-256.1)$ weeks in the ruxolitinib crossover group. The median (range) duration of exposure to placebo was 37.1 (3.6-65.3) weeks. Among patients who remained on treatment until study termination, the ruxolitinib exposure duration was 265.4 (249.9-296.0) weeks for the 43 patients in the group randomized to ruxolitinib and 229.6 (200.1-256.1) weeks for the 28 patients in the ruxolitinib crossover group. Ruxolitinib exposure was more than 1 to 2 years for $23 / 155$ (14.8\%) and 15/111 (13.5\%) patients in the ruxolitinib-randomized group and the ruxolitinib crossover group, respectively; more than 2 to 3 years for $22 / 155(14.2 \%)$ and $16 / 111$ (14.4\%) patients; more than 3 to 4 years for $19 / 155$ (12.3\%) and $11 / 111$ (9.9\%) patients; and more than 4 years for $62 / 155$ (40.0\%) and 35/111 (31.5\%) patients.

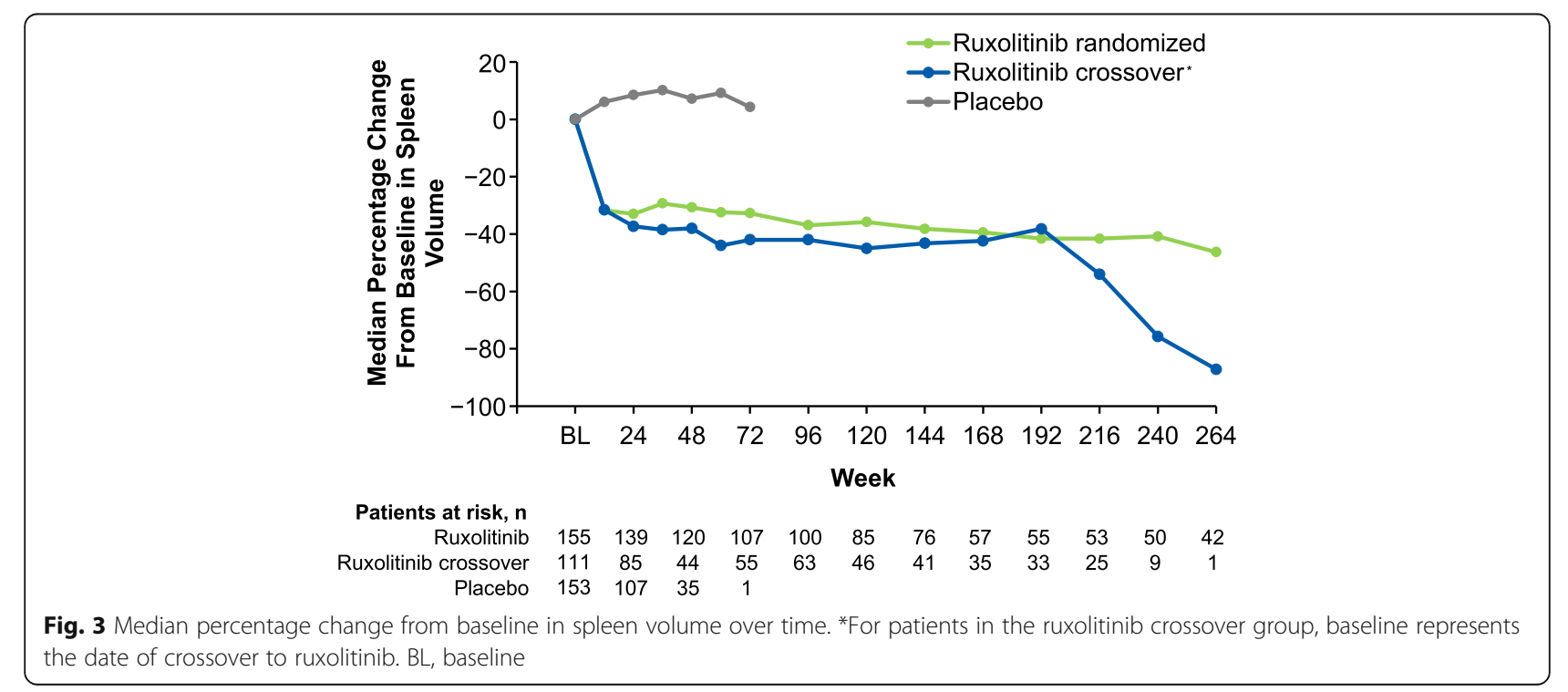




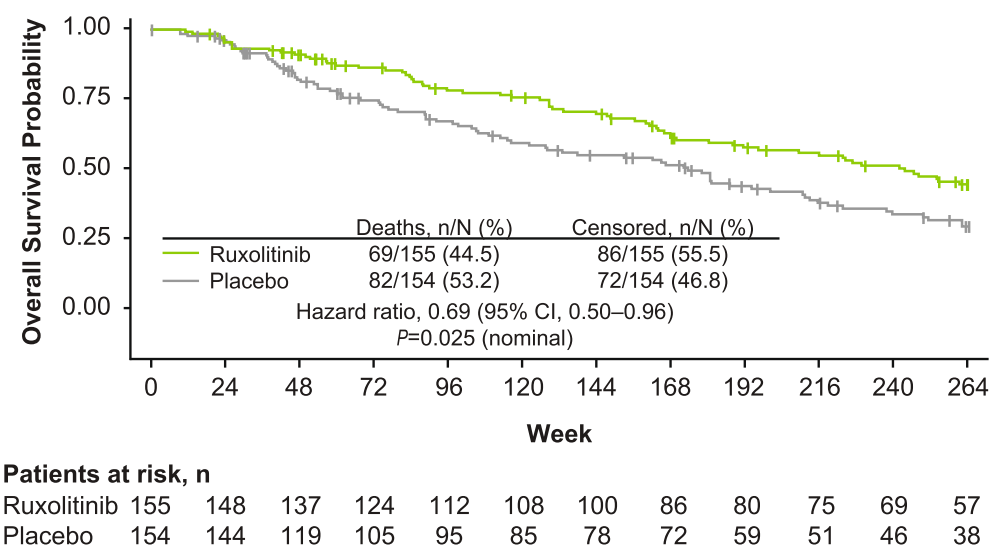

Fig. 4 Overall survival. The overall survival analysis included all patients who died during the study or during long-term follow-up after discontinuation of study treatment. HR, hazard ratio

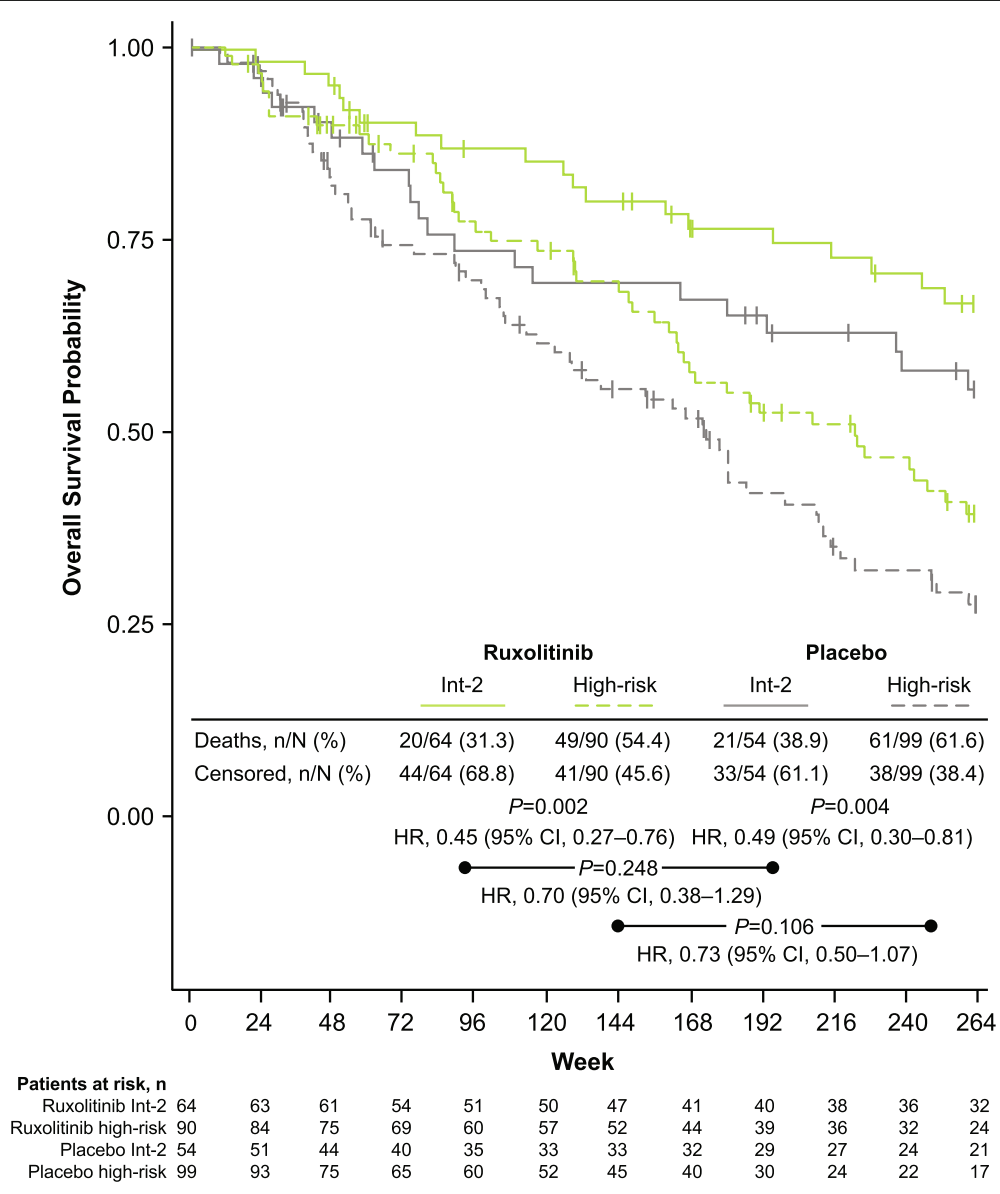

Fig. 5 Overall survival by IPSS risk status. In both treatment arms, overall survival was significantly longer for patients with int-2 compared with high-risk MF at diagnosis (ruxolitinib, $P=0.002$; placebo, $P=0.004$ ). Ruxolitinib was associated with nonsignificant survival advantages compared with placebo for both the int-2 and high-risk patient subgroups. HR, hazard ratio; int-2, intermediate-2; IPSS, International Prognostic Scoring System; MF, myelofibrosis 
The incidence of new-onset nonhematologic adverse events generally stabilized or decreased with long-term treatment in the ruxolitinib-randomized group (Tables 1 and 2). The most common new-onset all-grade nonhematologic adverse events starting $\geq 48$ months after ruxolitinib initiation were fatigue (33.3\%), pneumonia (16.4\%), constipation (16.0\%), cough (15.4\%), and headache (15.4\%); the most common grade 3 or 4 adverse events were pneumonia (15.6\%), congestive cardiac failure (6.2\%), sepsis (6.2\%), and squamous cell carcinoma (6.2\%).

Anemia and thrombocytopenia (per abnormal hematologic laboratory values) occurred in most patients in the ruxolitinib-randomized group (98.7 and 83.9\%, respectively). The rates of new or worsening grade 3 or 4 anemia, thrombocytopenia, and leukopenia were the highest within the first 6 months of treatment, decreasing thereafter (Fig. 6). No patients in the ruxolitinibrandomized group had new or worsening grade 3 or 4 anemia, thrombocytopenia, or leukopenia after month 42 . In agreement with the hematologic laboratory abnormalities over time in the ruxolitinib-randomized group, mean hemoglobin levels decreased during the first 12 weeks of treatment with randomized or crossover ruxolitinib but increased toward baseline levels and stabilized thereafter (Fig. 7). Similarly, mean platelet and white blood cell counts also decreased during the first 12 weeks of treatment with ruxolitinib, after which they remained stable (Fig. 7). In agreement with these blood count dynamics, the mean $(\mathrm{SD})$ number of transfusions per month in the ruxolitinib group peaked between weeks 4 and 8 (1.2 [1.75]) then decreased steadily until weeks 36 to $48(0.7$ [1.35]), stabilizing thereafter.

Table 1 Incidence of new-onset all-grade nonhematologic adverse events in the ruxolitinib group regardless of causality, grouped by treatment time interval

\begin{tabular}{|c|c|c|c|c|c|}
\hline & \multicolumn{5}{|c|}{ Ruxolitinib $(n=155)$} \\
\hline & $0-<12$ Months & $12-<24$ Months & $24-<36$ Months & $36-<48$ Months & $\geq 48$ Month \\
\hline \multicolumn{6}{|l|}{ Event, ${ }^{*} \mathrm{n} / \mathrm{N}(\%)$} \\
\hline Fatigue & 43/148.5 (29.0) & 14/92.0 (15.2) & 10/65.5 (15.3) & $5 / 45.0(11.1)$ & 7/21.0 (33.3) \\
\hline Diarrhea & 41/147.5 (27.8) & 6/89.0 (6.7) & 7/65.0 (10.8) & 5/46.5 (10.8) & 3/20.5 (14.6) \\
\hline Ecchymosis & $31 / 146.0(21.2)$ & 10/96.0 (10.4) & 4/70.0 (5.7) & $1 / 55.0(1.8)$ & $1 / 25.0(4.0)$ \\
\hline Dyspnea & 28/146.0 (19.2) & 10/98.5 (10.2) & 2/70.0 (2.9) & $2 / 54.5(3.7)$ & $3 / 25.0(12.0)$ \\
\hline Dizziness & 26/144.0 (18.1) & 10/96.0 (10.4) & 2/66.5 (3.0) & $1 / 49.5(2.0)$ & $1 / 21.5(4.7)$ \\
\hline Pain in extremity & 26/144.5 (18.0) & $6 / 97.0(6.2)$ & $3 / 71.0(4.2)$ & 2/51.5 (3.9) & $1 / 21.5(4.7)$ \\
\hline Peripheral edema & 26/145.5 (17.9) & $7 / 99.5(7.0)$ & 8/75.0 (10.7) & 3/53.5 (5.6) & $2 / 23.0(8.7)$ \\
\hline Headache & 24/144.5 (16.6) & $5 / 99.0(5.1)$ & $3 / 75.0(4.0)$ & $4 / 58.0(6.9)$ & $4 / 26.0(15.4)$ \\
\hline Nausea & 24/144.5 (16.6) & 7/102.5 (6.8) & 4/79.0 (5.1) & $5 / 61.0(8.2)$ & $4 / 27.5(14.5)$ \\
\hline Constipation & $21 / 145.0(14.5)$ & 10/105.0 (9.5) & 8/78.5 (10.2) & $4 / 56.5(7.1)$ & $4 / 25.0(16.0)$ \\
\hline Abdominal pain & 20/144.5 (13.8) & $6 / 106.0(5.7)$ & 3/84.0 (3.6) & $4 / 66.0(6.1)$ & $4 / 29.5(13.6)$ \\
\hline Insomnia & 20/144.5 (13.8) & 7/104.5 (6.7) & 3/80.0 (3.8) & $1 / 62.5(1.6)$ & $1 / 28.0(3.6)$ \\
\hline Vomiting & 20/145.5 (13.7) & 3/105.5 (2.8) & $2 / 82.5(2.4)$ & $4 / 64.5(6.2)$ & 4/29.0 (13.8) \\
\hline Pyrexia & 20/148.0 (13.5) & 8/109.5 (7.3) & $7 / 82.5(8.5)$ & 3/62.0 (4.8) & $2 / 27.5(7.3)$ \\
\hline Cough & 19/145.0 (13.1) & 14/105.5 (13.3) & $3 / 74.5(4.0)$ & $4 / 58.5(6.8)$ & $4 / 26.0(15.4)$ \\
\hline Arthralgia & 17/144.0 (11.8) & 6/103.0 (5.8) & $6 / 75.5(7.9)$ & 6/53.5 (11.2) & $3 / 21.5(14.0)$ \\
\hline Muscle spasms & 14/143.0 (9.8) & $3 / 105.0(2.9)$ & $7 / 81.0(8.6)$ & $6 / 58.0(10.3)$ & $1 / 23.0(4.3)$ \\
\hline Back pain & 13/143.0 (9.1) & 11/106.5 (10.3) & 0 & $4 / 58.0(6.9)$ & $3 / 25.5(11.8)$ \\
\hline Night sweats & 13/143.0 (9.1) & $3 / 105.5(2.8)$ & $3 / 81.5(3.7)$ & 1/61.5 (1.6) & $4 / 28.0(14.3)$ \\
\hline Pneumonia & 13/145.0 (9.0) & $7 / 110.0(6.4)$ & 3/82.5 (3.6) & 3/65.0 (4.6) & $5 / 30.5(16.4)$ \\
\hline Upper respiratory tract infection & $11 / 143.0(7.7)$ & 12/108.0 (11.1) & $4 / 74.5(5.4)$ & $4 / 55.0(7.3)$ & $3 / 24.0(12.5)$ \\
\hline Fall & $7 / 143.5(4.9)$ & 2/111.5 (1.8) & $1 / 87.0(1.1)$ & $3 / 68.5(4.4)$ & $4 / 30.5(13.1)$ \\
\hline Musculoskeletal pain & 7/143.0 (4.9) & $5 / 112.0(4.5)$ & $7 / 85.5(8.2)$ & $2 / 62.5(3.2)$ & $4 / 29.0(13.8)$ \\
\hline Pruritus & $7 / 142.5(4.9)$ & $8 / 110.5(7.2)$ & 1/81.0 (1.2) & 1/63.5 (1.6) & 3/29.0 (10.3) \\
\hline Herpes zoster & 3/143.5 (2.1) & $4 / 115.5(3.5)$ & $3 / 87.5(3.4)$ & $3 / 66.0(4.5)$ & 3/29.0 (10.3) \\
\hline Squamous cell carcinoma & 0 & $1 / 116.5(0.9)$ & 2/91.5 (2.2) & 2/70.5 (2.8) & $4 / 32.0(12.5)$ \\
\hline
\end{tabular}

*Occurring in $>10 \%$ of patients in the ruxolitinib group in $\geq 1$ yearly interval 
Table 2 Incidence of new-onset grade 3 or 4 nonhematologic adverse events in the ruxolitinib group regardless of causality, grouped by treatment time interval

\begin{tabular}{|c|c|c|c|c|c|}
\hline & \multicolumn{5}{|c|}{ Ruxolitinib $(n=155)$} \\
\hline & $0-<12$ Months & $12-<24$ Months & $24-<36$ Months & $36-<48$ Months & $\geq 48$ Months \\
\hline \multicolumn{6}{|l|}{ Event, ${ }^{*} \mathrm{n} / \mathrm{N}(\%)$} \\
\hline Fatigue & 9/144.5 (6.2) & 1/113.0 (0.9) & 3/90.0 (3.3) & $1 / 69.5(1.4)$ & 0 \\
\hline Pneumonia & 8/144.0 (5.6) & 4/112.0 (3.6) & $3 / 86.0(3.5)$ & $2 / 67.5(3.0)$ & $5 / 32.0(15.6)$ \\
\hline Abdominal pain & 6/143.5 (4.2) & 0 & $3 / 93.5(3.2)$ & $1 / 72.5(1.4)$ & $1 / 32.0(3.1)$ \\
\hline Arthralgia & 3/142.5 (2.1) & 0 & 0 & 1/70.0 (1.4) & 0 \\
\hline Diarrhea & 3/143.5 (2.1) & 0 & 0 & $1 / 72.5(1.4)$ & 0 \\
\hline Dyspnea & 3/143.5 (2.1) & $1 / 116.5(0.9)$ & $2 / 92.5(2.2)$ & $1 / 71.5(1.4)$ & $1 / 31.5(3.2)$ \\
\hline Pain in extremity & 3/142.5 (2.1) & 0 & $1 / 89.5(1.1)$ & $1 / 69.5(1.4)$ & $1 / 30.5(3.3)$ \\
\hline Acute myeloid leukemia & 2/143.5 (1.4) & 0 & $1 / 93.0(1.1)$ & $2 / 74.0(2.7)$ & 0 \\
\hline Fall & $2 / 142.5(1.4)$ & $1 / 114.5(0.9)$ & 0 & 2/71.0 (2.8) & $1 / 30.5(3.3)$ \\
\hline Gastrointestinal hemorrhage & $2 / 142.5(1.4)$ & 1/115.0 (0.9) & 0 & 0 & 0 \\
\hline Hyperuricemia & $2 / 142.5(1.4)$ & 1/114.5 (0.9) & 0 & $1 / 71.5(1.4)$ & 0 \\
\hline Hypoxia & $2 / 142.5(1.4)$ & 0 & $2 / 92.0(2.2)$ & 0 & $1 / 31.5(3.2)$ \\
\hline Muscular weakness & 2/143.0 (1.4) & 0 & $1 / 91.5(1.1)$ & 0 & 0 \\
\hline Septic shock & 2/143.5 (1.4) & 0 & 0 & 0 & 0 \\
\hline Acute renal failure & $1 / 142.5(0.7)$ & 1/116.0 (0.9) & 3/93.0 (3.2) & 2/72.5 (2.8) & $1 / 31.5(3.2)$ \\
\hline Back pain & $1 / 142.5(0.7)$ & $2 / 116.0(1.7)$ & 0 & 0 & 0 \\
\hline Congestive cardiac failure & $1 / 142.5(0.7)$ & 0 & $1 / 92.0(1.1)$ & 0 & $2 / 32.5(6.2)$ \\
\hline Epistaxis & 1/143.0 (0.7) & $2 / 117.0(1.7)$ & 0 & 0 & 0 \\
\hline Sepsis & $1 / 143.0(0.7)$ & $2 / 116.5(1.7)$ & $2 / 92.5(2.2)$ & 1/73.0 (1.4) & $2 / 32.5(6.2)$ \\
\hline Upper abdominal pain & $1 / 143.0(0.7)$ & 0 & $2 / 92.5(2.2)$ & 0 & 0 \\
\hline Cellulitis & 0 & 0 & 0 & $2 / 73.5(2.7)$ & 0 \\
\hline Myocardial infarction & 0 & 1/117.0 (0.9) & 0 & $2 / 73.5(2.7)$ & 0 \\
\hline Osteoarthritis & 0 & 0 & $1 / 92.5(1.1)$ & 0 & $2 / 32.5(6.2)$ \\
\hline Osteomyelitis & 0 & 0 & 0 & $2 / 73.0(2.7)$ & 0 \\
\hline Squamous cell carcinoma & 0 & 1/116.5 (0.9) & 0 & 0 & $2 / 32.5(6.2)$ \\
\hline Urinary tract infection & 0 & 1/116.5 (0.9) & $1 / 92.0(1.1)$ & 0 & $2 / 33.0(6.1)$ \\
\hline Wound infection & 0 & 0 & 0 & 0 & $2 / 33.0(6.1)$ \\
\hline
\end{tabular}

*Occurring in $\geq 2$ patients in the ruxolitinib group in any yearly interval

Serious adverse events occurred at any time during treatment with ruxolitinib in 103/155 (66.5\%) patients in the ruxolitinib-randomized group and 74/111 (66.7\%) patients in the ruxolitinib crossover group. The most frequent serious adverse events, occurring in $\geq 4 \%$ of patients in the ruxolitinib-randomized or crossover groups, were pneumonia (randomized, 15.5\%; crossover, 10.8\%), anemia $(11.0 \% ; 11.7 \%)$, sepsis $(4.5 \% ; 3.6 \%)$, and congestive cardiac failure $(3.2 \% ; 4.5 \%)$.

Throughout the course of the study, adverse events resulted in a ruxolitinib dose decrease in 88/155 (56.8\%) patients in the ruxolitinib-randomized group and 45/111 (40.5\%) patients in the ruxolitinib crossover group. Thrombocytopenia was the most frequent cause of dose decreases, occurring in 75/155 (48.4\%) and 36/111
(32.4\%) patients in the ruxolitinib-randomized and crossover groups, respectively.

Adverse events resulted in discontinuation of treatment in 50/155 (32.3\%) patients originally randomized to ruxolitinib, 39/111 (35.1\%) in the ruxolitinib crossover group, and 19/151 (12.6\%) during treatment with placebo. The most common reasons, occurring in $\geq 2.0 \%$ of patients treated with ruxolitinib, were disease progression $(3.2 \%)$, acute myeloid leukemia (AML; $2.6 \%$ ), and anemia $(2.6 \%)$ in the ruxolitinib-randomized group, and thrombocytopenia (3.6\%) and AML (3.6\%) in the ruxolitinib crossover group.

Herpes zoster infections occurred at higher rates in patients treated with ruxolitinib compared with placebo (Table 3). All herpes zoster events in the ruxolitinib- 


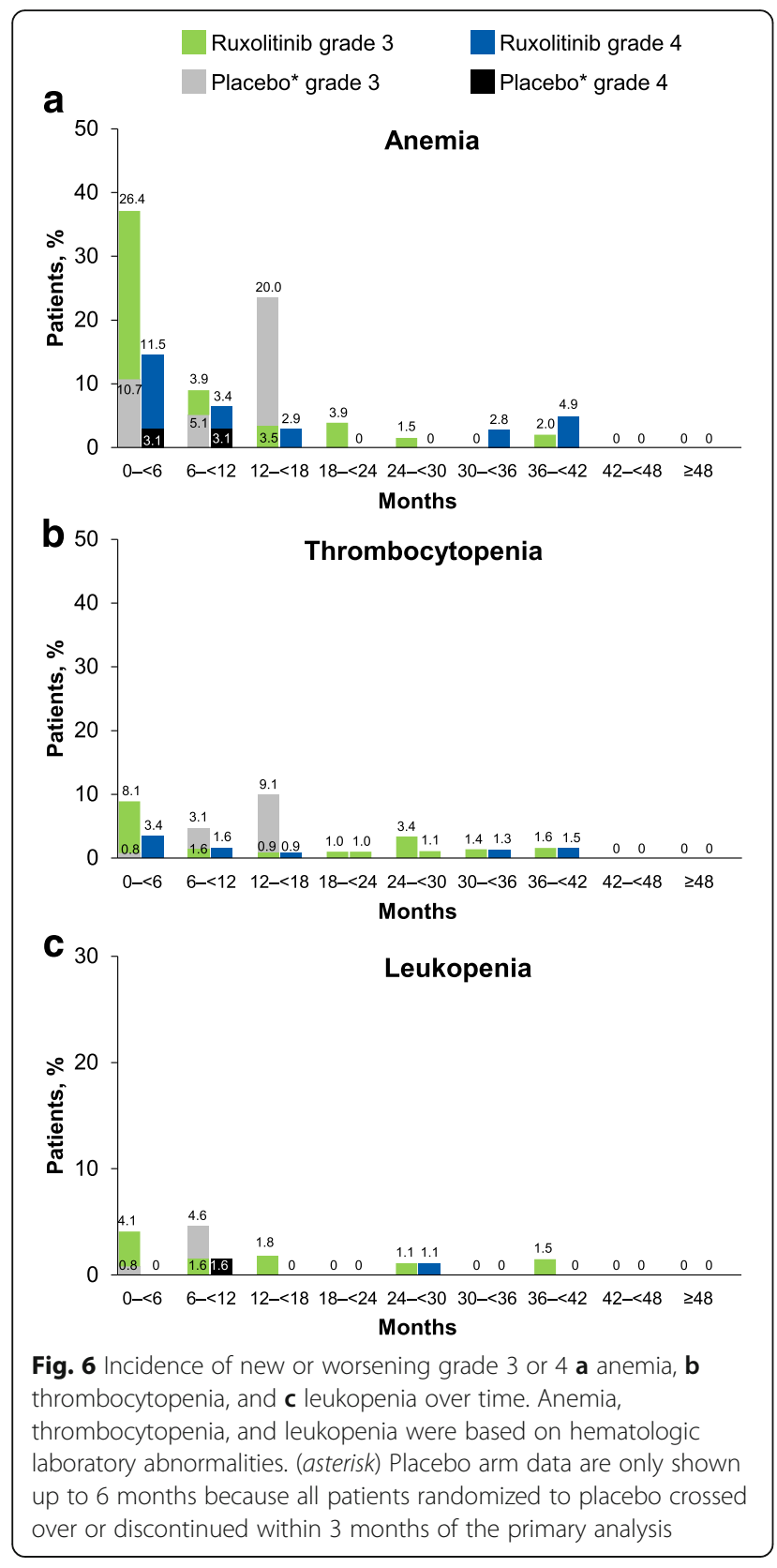

randomized group were grade 1 or 2, occurring in three or four patients each year. Among all patients treated with ruxolitinib, the majority of cases were single episodes that were grade 2 or lower and resolved without long-term sequelae. The only serious event of herpes zoster infection occurred in a patient randomized to placebo after crossing over to ruxolitinib.

Sepsis occurred at similar rates between patients treated with ruxolitinib and those receiving placebo (Table 3). All sepsis events were grade 3 or 4 , with the exception of one grade 2 event in the placebo group. Serious events of sepsis and septic shock occurred at

\section{a}

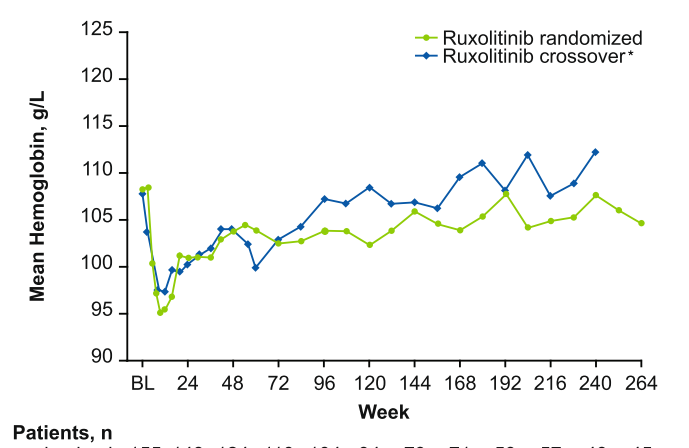

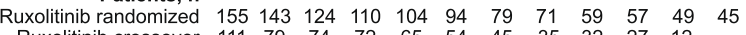
$\begin{array}{llllllllllll}\text { Ruxolitinib crossover } & 111 & 79 & 74 & 72 & 65 & 54 & 45 & 35 & 32 & 27 & 12\end{array}$

b

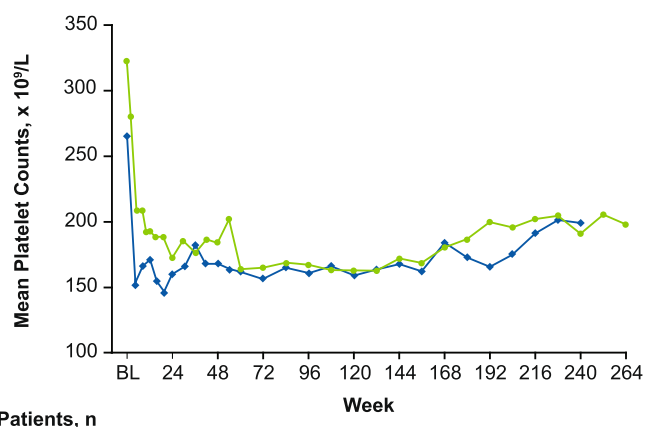

$\begin{array}{lllllllllllll}\text { Ruxolitinib randomized } & 155 & 143 & 124 & 110 & 104 & 94 & 79 & 71 & 59 & 57 & 49 & 45\end{array}$ $\begin{array}{llllllllllll}\text { Ruxolitinib crossover } & 111 & 79 & 74 & 72 & 64 & 54 & 45 & 35 & 32 & 26 & 12\end{array}$

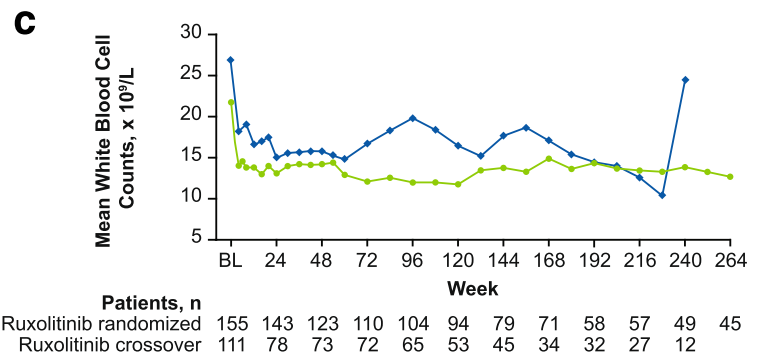

Fig. 7 Mean blood counts over time in the ruxolitinib randomized and ruxolitinib crossover groups. Blood counts were based on measurements of a hemoglobin level, $\mathbf{b}$ platelet counts, and $\mathbf{c}$ white blood cell counts. *For patients in the ruxolitinib crossover group, BL represents the date of crossover to ruxolitinib. $\mathrm{BL}$, baseline

rates of 1.5 and 0.4 per 100 patient-years of exposure in the ruxolitinib-randomized group and 1.5 each in the ruxolitinib crossover group.

Nonmelanoma skin cancers, including basal cell carcinoma and squamous cell carcinoma of the skin, occurred at similar rates between patients treated with ruxolitinib and those receiving placebo (Table 3). Basal cell carcinoma occurred at a rate of 2.7 per 100 patientyears of exposure in the ruxolitinib-randomized group, 4.0 in the ruxolitinib crossover group, and 3.9 among patients during treatment with placebo (Table 3). There were two cases of basal cell carcinoma in the ruxolitinib crossover group; in both cases, patients had a history of skin cancer. 
Table 3 Exposure-adjusted rates of select adverse events

\begin{tabular}{|c|c|c|c|c|c|c|}
\hline \multirow[t]{2}{*}{ Median duration of exposure, $d$} & \multicolumn{2}{|c|}{$\begin{array}{l}\text { Ruxolitinib randomized } \\
(n=155) \\
1045.0\end{array}$} & \multicolumn{2}{|c|}{$\begin{array}{l}\text { Ruxolitinib crossover } \\
(n=111)^{*} \\
777.0\end{array}$} & \multicolumn{2}{|c|}{$\begin{array}{l}\text { During placebo treatment } \\
(n=151)^{*} \\
260.0\end{array}$} \\
\hline & All grade & Grade 3 or 4 & All grade & Grade 3 or 4 & All grade & Grade 3 or 4 \\
\hline \multicolumn{7}{|l|}{ Event, n/PYE (rate per 100 PYE) } \\
\hline \multicolumn{7}{|l|}{ Infections and infestations ${ }^{a}$} \\
\hline Upper respiratory tract infection & $34 / 398.0(8.5)$ & 0 & $22 / 230.5(9.5)$ & 0 & 15/96.9 (15.5) & $1 / 96.9(1.0)$ \\
\hline Urinary tract infection & $31 / 414.3(7.5)$ & 4/414.3 (1.0) & $16 / 240.5(6.7)$ & $3 / 240.5(1.2)$ & 7/101.3 (6.9) & 1/101.3 (1.0) \\
\hline Pneumonia & $31 / 432.3(7.2)$ & $22 / 432.3(5.1)$ & 18/253.6 (7.1) & 8/253.6 (3.2) & 11/102.4 (10.7) & 8/102.4 (7.8) \\
\hline Herpes zoster & $16 / 452.5(3.5)$ & 0 & $14 / 241.2(5.8)$ & 1/242.2 (0.4) & $1 / 104.1(1.0)$ & 0 \\
\hline Bronchitis & $14 / 450.5(3.1)$ & 0 & $11 / 244.9(4.5)$ & $3 / 244.9(1.2)$ & 2/104.2 (1.9) & 0 \\
\hline Nasopharyngitis & 14/449.1 (3.1) & 0 & 9/253.6 (3.5) & 0 & 9/98.4 (9.1) & 0 \\
\hline Sinusitis & 12/453.2 (2.6) & $1 / 453.2(0.2)$ & 7/252.3 (2.8) & 0 & 3/102.7 (2.9) & $1 / 102.7(1.0)$ \\
\hline Cellulitis & 10/467.8 (2.1) & 2/467.8 (0.4) & $3 / 262.6(1.1)$ & 0 & 2/103.5 (1.9) & 0 \\
\hline Influenza & $8 / 469.0(1.7)$ & 0 & $3 / 266.0(1.1)$ & $1 / 266.0(0.4)$ & 0 & 0 \\
\hline Sepsis & $8 / 480.3(1.7)$ & 8/480.3 (1.7) & 4/267.4 (1.5) & $4 / 267.4(1.5)$ & 2/104.0 (1.9) & 1/104.0 (1.0) \\
\hline Tooth abscess & $7 / 476.3(1.5)$ & $1 / 476.3(0.2)$ & $4 / 261.3(1.5)$ & 0 & 0 & 0 \\
\hline Oral herpes & 6/469.8 (1.3) & 0 & 2/269.1 (0.7) & 0 & 2/103.2 (1.9) & 0 \\
\hline Skin infection & $5 / 469.8(1.1)$ & 0 & 3/269.5 (1.1) & 0 & 1/104.4 (1.0) & 0 \\
\hline Viral infection & $5 / 471.2(1.1)$ & 0 & 2/265.4 (0.8) & 0 & 0 & 0 \\
\hline Viral gastroenteritis & 4/470.6 (0.9) & 0 & 1/270.1 (0.4) & 0 & 2/103.5 (1.9) & 0 \\
\hline Diverticulitis & 4/475.0 (0.8) & 1/475.0 (0.2) & 3/268.5 (1.1) & 1/268.5 (0.4) & 2/103.6 (1.9) & 0 \\
\hline Ear infection & 4/473.3 (0.8) & 0 & $4 / 267.8(1.5)$ & 0 & 0 & 0 \\
\hline Fungal infection & 4/479.4 (0.8) & 0 & 2/267.6 (0.7) & 1/267.6 (0.4) & 2/103.8 (1.9) & 0 \\
\hline Localized infection & 4/479.1 (0.8) & 0 & 1/269.2 (0.4) & 1/269.2 (0.4) & 1/104.1 (1.0) & 0 \\
\hline Lower respiratory tract infection & 4/476.9 (0.8) & 0 & 1/270.5 (0.4) & 0 & 2/103.3 (1.9) & 1/103.3 (1.0) \\
\hline Septic shock & 2/484.6 (0.4) & 2/484.6 (0.4) & 3/270.5 (1.1) & $3 / 270.5(1.1)$ & 0 & 0 \\
\hline \multicolumn{7}{|l|}{ Neoplasms } \\
\hline Basal cell carcinoma & $12 / 450.9(2.7)$ & $2 / 450.9(0.4)$ & 10/252.7 (4.0) & 2/252.7 (0.8) & 4/103.7 (3.9) & 0 \\
\hline Squamous cell carcinoma & $10 / 462.6(2.2)$ & $2 / 462.6(0.8)$ & 10/252.0 (4.0) & $3 / 252.0(1.2)$ & 4/102.9 (3.9) & 0 \\
\hline Squamous cell carcinoma of the skin & 9/470.2 (1.9) & 3/470.2 (0.6) & $3 / 266.4(1.1)$ & $1 / 266.4(0.4)$ & $1 / 104.7(1.0)$ & 0 \\
\hline Acute myeloid leukemia & $5 / 483.8(1.0)$ & $5 / 483.8(1.0)$ & 5/270.1 (1.9) & $5 / 270.1(1.9)$ & 0 & 0 \\
\hline
\end{tabular}

PYE, patient-years of exposure

*Adverse events that occurred following the first dose of ruxolitinib (ie, after crossover from placebo) were included in the ruxolitinib crossover group ${ }^{a}$ Occurring in $\geq 5$ patients treated with ruxolitinib

Disease transformation to AML occurred in five patients each in the ruxolitinib-randomized and ruxolitinib crossover groups; no patients developed AML during treatment with placebo (Table 3). Overall, AML occurred in five male and five female patients. The median (range) time from the first ruxolitinib dose to AML diagnosis was 838 (157-1150) days in the ruxolitinib-randomized group and 376 (21-666) days in the ruxolitinib crossover group; median (range) time from MF diagnosis to AML diagnosis was 1190 (699-1708) days and 1015 (372-11,971) days, respectively. Prior medications for the treatment of MF in patients who developed AML were hydroxyurea (ruxolitinib-randomized group, $n=2$; ruxolitinib crossover group, $n=2$ ) and lenalidomide, and corticosteroids (all in one patient in the ruxolitinib crossover group); three patients in the ruxolitinibrandomized group and two in the ruxolitinib crossover group had no prior treatments for MF.

Overall, 28/155 (18.1\%) patients in the ruxolitinibrandomized group and 39/151 (25.8\%) in the placebo randomized group experienced a treatment-emergent adverse event that resulted in death while on study or within 28 days of the last dose of study drug. Among the patients randomized to placebo, a treatment-emergent adverse event led to death in 11/151 (7.3\%) patients 
Table 4 Treatment-emergent adverse events resulting in death*

\begin{tabular}{|c|c|c|c|}
\hline Cause of death, $n(\%)^{a}$ & $\begin{array}{l}\text { Ruxolitinib } \\
\text { randomized } \\
(n=155)\end{array}$ & $\begin{array}{l}\text { After ruxolitinib } \\
\text { crossover }^{\mathrm{b}} \\
(n=111)\end{array}$ & $\begin{array}{l}\text { During placebo } \\
\text { treatment } \\
(n=151)\end{array}$ \\
\hline $\begin{array}{l}\text { Death caused by any treatment- } \\
\text { emergent adverse event }\end{array}$ & $28(18.1)$ & $28(25.2)$ & $11(7.3)$ \\
\hline Sepsis & $4(2.6)$ & $2(1.8)$ & $1(0.7)$ \\
\hline Disease progression & $3(1.9)$ & $4(3.6)$ & $3(2.0)$ \\
\hline Pneumonia & $3(1.9)$ & $1(0.9)$ & $1(0.7)$ \\
\hline Acute myeloid leukemia & $2(1.3)$ & $3(2.7)$ & 0 \\
\hline Cerebral hemorrhage & $2(1.3)$ & $1(0.9)$ & $1(0.7)$ \\
\hline Septic shock & $2(1.3)$ & $2(1.8)$ & 0 \\
\hline Acute renal failure & $1(0.6)$ & $1(0.9)$ & 0 \\
\hline Anemia & $1(0.6)$ & 0 & 0 \\
\hline Cardiac arrest & $1(0.6)$ & 0 & 0 \\
\hline Death, unspecified & $1(0.6)$ & $1(0.9)$ & 0 \\
\hline Falling injury & $1(0.6)$ & 0 & 0 \\
\hline Hemorrhagic shock & $1(0.6)$ & $1(0.9)$ & 0 \\
\hline Metastatic NSCLC & $1(0.6)$ & 0 & 0 \\
\hline Multiorgan failure & $1(0.6)$ & 0 & $1(0.7)$ \\
\hline Muscular weakness & $1(0.6)$ & 0 & 0 \\
\hline Myocardial infarction & $1(0.6)$ & $1(0.9)$ & 0 \\
\hline Pancreatic carcinoma & $1(0.6)$ & 0 & 0 \\
\hline Renal failure & $1(0.6)$ & 0 & 0 \\
\hline Respiratory failure & $1(0.6)$ & 0 & 0 \\
\hline Splenic infarction & $1(0.6)$ & 0 & 0 \\
\hline Congestive cardiac failure & 0 & $2(1.8)$ & 0 \\
\hline Myelofibrosis & 0 & $2(1.8)$ & $1(0.7)$ \\
\hline Cardiac failure & 0 & $1(0.9)$ & 0 \\
\hline Pneumonia aspiration & 0 & $2(1.8)$ & 0 \\
\hline Anastomotic hemorrhage & 0 & $1(0.9)$ & 0 \\
\hline Cholecystitis & 0 & $1(0.9)$ & 0 \\
\hline Delirium & 0 & $1(0.9)$ & 0 \\
\hline Road traffic accident & 0 & $1(0.9)$ & 0 \\
\hline Splenic rupture & 0 & $1(0.9)$ & 0 \\
\hline Suicide & 0 & $1(0.9)$ & 0 \\
\hline Gastrointestinal hemorrhage & 0 & 0 & $1(0.7)$ \\
\hline Intestinal perforation & 0 & 0 & $1(0.7)$ \\
\hline Staphylococcal infection & 0 & 0 & $1(0.7)$ \\
\hline
\end{tabular}

NSCLC, non-small cell lung cancer

*Limited to fatal treatment-emergent adverse events occurring during treatment with study drug or within 28 days of the last dose of study drug a Patient deaths were counted once under each Medical Dictionary for Regulatory Activities system organ class and preferred term, and therefore individual patients may have had $>1$ cause of death

${ }^{b}$ Fatal treatment-emergent adverse events that occurred following the first dose of ruxolitinib (ie, after crossover from placebo) were included in the ruxolitinib crossover group

during treatment with placebo and 28/111 (25.2\%) patients after crossover to ruxolitinib (Table 4).

\section{Discussion}

This final analysis of the COMFORT-I trial demonstrated that treatment with ruxolitinib was associated with rapid and durable reductions in splenomegaly and significantly longer OS compared with patients originally randomized to placebo. Patient risk of death was approximately 30\% lower in the ruxolitinib group compared with placebo, despite the crossover from placebo to ruxolitinib. Given that COMFORT-I was restricted to patients with intermediate-2 or high-risk MF with splenomegaly, OS data suggest that delaying treatment with ruxolitinib may worsen outcomes and that studies evaluating ruxolitinib in patients with earlier MF disease states may be warranted.

The exact mechanism by which ruxolitinib prolongs survival and ameliorates splenomegaly remains unclear, but it is rational to hypothesize that the downstream effects of ruxolitinib confer changes in cytokines, metabolic properties, and JAK2V617F allele burden that may play a role. Ruxolitinib has been associated with reductions in inflammatory cytokines and markers of inflammation [12], improvements in measures of metabolic and nutrition status [20], reduced fibrosis in some patients [17], and reductions in JAK2V617F allele burden [21]. In COMFORT-I patients receiving long-term treatment with ruxolitinib, relationships have been identified between reductions in spleen volume and (1) increases in body weight and normalization of serum albumin and total cholesterol levels [20] and (2) reductions in $J A K 2 \mathrm{~V} 617 \mathrm{~F}$ allele burden in some patients [21]. In addition, ruxolitinib has been associated with improvements in spleen volume and OS in a wide variety of patient subgroups stratified by MF subtype, age, IPSS risk score, ECOG performance status, and baseline hemoglobin level, platelet count, spleen size, and $J A K 2$ V617F mutation status [14]. Future work will be required to elucidate the mechanism by which ruxolitinib is efficacious and if there are any related disease markers or patient characteristics that could be helpful in identifying the types of patients who may benefit the most from ruxolitinib treatment.

Overall, the safety profile was supportive of long-term treatment with ruxolitinib, with no unexpected safety signals. The nonhematologic adverse event rates generally remained stable or decreased with prolonged ruxolitinib treatment duration and were consistent with those reported in previous analyses of the COMFORT-I study $[12,15]$. As expected, based on the mechanism of action of ruxolitinib as a JAK1/JAK2 inhibitor [22, 23], thrombocytopenia and anemia occurred in most patients treated with ruxolitinib. Anemia and thrombocytopenia can be managed with dose adjustments and, for some patients with anemia, red blood cell transfusions [24]. Indeed, although thrombocytopenia was the most common cause for ruxolitinib dose reduction in COMFORT-I, thrombocytopenia and anemia resulted in relatively few discontinuations (each $\leq 3.6 \%$ in the ruxolitinib- 
randomized and crossover groups). Mean hemoglobin, platelet, and white blood cell levels stabilized after 12 weeks of treatment, with hemoglobin levels returning to near-baseline levels thereafter; however, this finding must be interpreted taking into account the positive selection of patients remaining on study. Mean blood transfusion rates were in agreement with these trends. Nevertheless, ruxolitinib may provide a survival benefit even in the presence of anemia. In a pooled analysis of COMFORT-I and COMFORT-II 3year data, treatment with ruxolitinib was associated with a survival advantage regardless of anemia at baseline (3-year OS probability: ruxolitinib, 0.66; control, 0.57) or after initiating study treatment (3-year OS probability: ruxolitinib, 0.87; control, 0.66) [25].

Herpes zoster infections occurred at higher rates among patients treated with ruxolitinib compared with placebo. The incidence of herpes zoster infections increased with longer exposure to ruxolitinib (0-12 months' exposure, $2.1 \%$; $\geq 48$ months' exposure, $10.3 \%$ ). However, all but one case was grade 1 or 2 , and it is unclear if this increase was clinically relevant. Other infections, including pneumonia, sepsis, upper respiratory tract infection, and urinary tract infection, occurred at similar or lower rates with ruxolitinib compared with placebo; however, pneumonia was the most common new-onset grade 3 or 4 adverse event observed after 48 months of treatment with ruxolitinib. Nonmelanoma skin cancers were observed in patients treated with ruxolitinib; however, these occurred at rates that were similar to or lower than those observed during treatment with placebo. Finally, the incidence of AML transformation in the ruxolitinib-randomized and crossover groups was consistent with previous reports in patients with MF [26, 27]. Although no patients developed AML during treatment with placebo, the median exposure (37.1 weeks) may not have been long enough to observe AML transformations considering that the median time from the first ruxolitinib dose to AML diagnosis was 119.7 weeks in the ruxolitinib-randomized group.

Overall, $48.9 \%$ of the COMFORT-I patient population had died by the time of the final 5-year analysis. Causes of death were generally consistent with expected morbidities resulting from MF progression and/or other underlying disease processes (e.g., infections, transformation to AML), particularly in elderly and chronically ill patients. The most common adverse events leading to death in COMFORT-I were sepsis or septic shock, followed by disease progression, pneumonia, and transformation to AML. Eleven patients treated with ruxolitinib died because of a cardiovascular, thrombotic, or hemorrhagic event. In comparison, an international retrospective analysis of 1131 patients with PMF enrolled between 1980 and 2007 reported that the leading causes of death were transformation to AML, disease progression, thrombosis and cardiovascular complications, and infection [19].

\section{Conclusions}

This final analysis of the COMFORT-I study included 5 years of treatment duration and demonstrated that long-term ruxolitinib treatment in patients with intermediate- 2 or high-risk MF was associated with durable reductions in spleen size and significantly longer OS compared with placebo. The safety profile continued to remain consistent with previous COMFORT-I and COMFORT-II analysis [12, 13, 15-17], with no new or unexpected adverse events identified with long-term treatment. Collectively, these data and similar findings in the 5-year analysis of the COMFORT-II study [17] support ruxolitinib as an effective long-term treatment option for patients with intermediate- 2 or high-risk MF.

\section{Abbreviations \\ AML: Acute myeloid leukemia; BL: Baseline; COMFORT: Controlled Myelofibrosis Study with Oral JAK Inhibitor Treatment; ECOG: Eastern Cooperative Oncology Group; HR: Hazard ratio; IPSS: International Prognostic Scoring System; IVRS: Interactive voice response system; JAK: Janus kinase; MF: Myelofibrosis; NE: Not evaluable; NSCLC: Non-small cell lung cancer; OS: Overall survival; PET-MF: Post-essential thrombocythemia myelofibrosis; PMF: Primary myelofibrosis; PPV-MF: Post-polycythemia vera myelofibrosis; PV: Polycythemia vera; PYE: Patient-years exposure; QoL: Quality of life}

\section{Acknowledgements}

Writing assistance was provided by Cory Pfeiffenberger, PhD (Complete Healthcare Communications, LLC, an ICON plc company), whose work was funded by Incyte Corporation.

\section{Funding}

This study was funded by Incyte Corporation.

\section{Availability of data and materials}

The datasets collected and/or analyzed during the current study are available from the corresponding author on reasonable request.

\section{Authors' contributions}

SV, RAM, JG, VG, JFD, JVC, MWND, CBM, RTS, MT, EFW, JHH, MOA, EOH, RML, $R P, A R$, and HK designed and performed the study. MJ, DK, and KS designed the study and analyzed the data. All authors participated in drafting the manuscript and approved the final version of the manuscript for submission.

\section{Competing interests}

SV participated in advisory boards for Incyte Corporation and received research support for the conduct of clinical studies from Incyte Corporation, Roche, AstraZeneca, Lilly Oncology, Geron, NS Pharma, Bristol-Myers Squibb, Celgene, Gilead, Seattle Genetics, Promedior, CTI BioPharma Corp, Galena BioPharma, Pfizer, and Genentech. RAM received research funding from Incyte Corporation, CTI, Gilead Sciences, Genentech, Eli Lilly, Promedior, NS Pharma, Sanofi, and Celgene and has served as a consultant for Novartis and ARIAD. JG received research funding from Incyte Corporation, Gilead Sciences, Novartis, Promedior, and CTI Biopharma. VG received honoraria and research funding from Incyte Corporation. JFD, JHH, EOH, and AR received research funding from Incyte Corporation. JVC received research funding from Incyte Corporation and served as a consultant for and received honoraria from Roche, Gilead Sciences, and Celgene Corporation. MWND served as a consultant for and received honoraria and research funding from Incyte Corporation. CBM received research funding and honoraria and served on a speakers bureau and as a consultant for Incyte Corporation, and received research funding and served as a consultant and on a speakers bureau for Novartis. RTS received research funding from Promedior and Incyte Corporation and served as a consultant to Incyte Corporation, Sanofi, PharmaEssentia, AOP 
Orphan, and Gilead Sciences. MT has received research funding from ARIAD, Bristol-Myers Squibb, Sanofi, Incyte Corporation, and Pfizer. EFW has received research funding from Gilead Sciences, Incyte Corporation, Pfizer, and Sanofi and has served on advisory boards for Incyte Corporation. MOA received research funding from Incyte Corporation and Gilead Sciences. RML received research funding from Galena Biopharma Incorporated and Incyte Corporation and served as a consultant for Amgen. RP received research funding from Incyte Corporation and received honoraria from ARIAD, Bristol-Myers Squibb, and Novartis. MJ, DK, and KS are employees and stockholders of Incyte Corporation. HK received research funding from ASTEX and Incyte Corporation.

\section{Consent for publication}

Not applicable.

\section{Ethics approval and consent to participate}

This study was conducted in accordance with the International Conference on Harmonization guidelines for Good Clinical Practice. The protocol was reviewed and approved by each participating site's institutional review board. All patients provided written informed consent prior to study participation.

\section{Previous presentations}

European Hematology Association Annual Meeting; June $9-12$ 2016; Copenhagen Denmark (abstract S452)

American Society of Clinical Oncology Annual Meeting; June 3-7; 2016; Chicago IL, USA (abstract 7012)

\section{Author details}

'Division of Cancer Medicine, The University of Texas MD Anderson Cancer Center, 1515 Holcombe Blvd, Unit 418, Houston, TX 77030, USA. ${ }^{2}$ Mayo Clinic Cancer Center, 13400 E. Shea Blvd, Scottsdale, AZ 85259, USA. ${ }^{3}$ Stanford Cancer Institute, 875 Blake Wilbur Drive, Room 2324, Stanford, CA 94305, USA. ${ }^{4}$ Princess Margaret Cancer Center, University of Toronto, 610 University Avenue, Suite 5-217, Toronto M5G 2M9, Canada. ${ }^{5}$ Washington University School of Medicine, 660S Euclid Ave., St. Louis, MO 63110, USA. ${ }^{6}$ Frankston Hospital and Department of Clinical Haematology, Monash University, 2 Hastings Rd, Frankston, VIC 3199, Australia. ${ }^{7}$ University of Utah Huntsman Cancer Institute, 2000 Circle of Hope, Salt Lake City, UT 84112, USA. ${ }^{8}$ Saint Agnes Cancer Institute, 9005 Caton Ave., Baltimore, MD 21229, USA. 'Weill Cornell Medical Center, 525 East 68th St., Payson Pavilion 3, New York, NY 10021, USA. ${ }^{10}$ University of Michigan, 1500 E Medical Center Dr., Ann Arbor, MI 48109, USA. ${ }^{11}$ Emory University School of Medicine, 1365 C Clifton Rd. Suite 1152, Atlanta, GA 30322, USA. ${ }^{2}$ Birmingham Hematology and Oncology, 1024 First St North, Birmingham, AL 35204, USA. ${ }^{13}$ Duke University Health System, DUMC 3912, Durham, NC 27710, USA. ${ }^{14}$ Abramson Cancer Center at the University of Pennsylvania, 3400 Civic Center Blvd., PCAM 2 West Pavilion, Philadelphia, PA 19104, USA. ${ }^{15}$ Texas Oncology and US Oncology Research, 4411 Medical Dr., San Antonio, TX 78229, USA. ${ }^{16}$ Cedars-Sinai, 10833 Le Conte Ave., 42-121 CHS, Los Angeles, CA 90024, USA. ${ }^{17}$ Columbia University Medical Center, Milstein Hospital Building, 6N-435, 177 Fort Washington Ave., New York, NY 10032, USA. ${ }^{18}$ Incyte Corporation, 1801 Augustine Cut-Off, Wilmington, DE 19803, USA. ${ }^{19}$ Division of Hematology and Hematologic Malignancies and Huntsman Cancer Institute, University of Utah, Salt Lake City, UT 84112, USA.

\section{Received: 14 December 2016 Accepted: 8 February 2017} Published online: 22 February 2017

\section{References}

1. Arber DA, Orazi A, Hasserjian R, Thiele J, Borowitz MJ, Le Beau MM, et al. The 2016 revision to the World Health Organization classification of myeloid neoplasms and acute leukemia. Blood. 2016;127:2391-405.

2. Mesa R, Miller CB, Thyne M, Mangan J, Goldberger S, Fazal S, et al. Myeloproliferative neoplasms (MPNs) have a significant impact on patients' overall health and productivity: the MPN Landmark survey. BMC Cancer. 2016;16:167

3. Pardanani A, Guglielmelli P, Lasho TL, Pancrazzi A, Finke CM, Vannucchi AM, et al. Primary myelofibrosis with or without mutant MPL: comparison of survival and clinical features involving 603 patients. Leukemia. 2011;25:1834-9.
4. Hultcrantz M, Kristinsson SY, Andersson TM, Landgren O, Eloranta S, Derolf AR, et al. Patterns of survival among patients with myeloproliferative neoplasms diagnosed in Sweden from 1973 to 2008: a population-based study. J Clin Oncol. 2012;30:2995-3001.

5. Gupta V, Hari P, Hoffman R. Allogeneic hematopoietic cell transplantation for myelofibrosis in the era of JAK inhibitors. Blood. 2012;120:1367-79.

6. Tefferi A, Guglielmelli P, Larson DR, Finke C, Wassie EA, Pieri L, et al. Long-term survival and blast transformation in molecularly-annotated essential thrombocythemia, polycythemia vera and myelofibrosis. Blood. 2014:124:2507-13.

7. Klampfl T, Gisslinger H, Harutyunyan AS, Nivarthi H, Rumi E, Milosevic JD, et al. Somatic mutations of calreticulin in myeloproliferative neoplasms. N Engl J Med. 2013;369:2379-90.

8. Guglielmelli P, Rotunno G, Fanelli T, Pacilli A, Brogi G, Calabresi L, et al. Validation of the differential prognostic impact of type 1/type 1-like versus type 2/type 2-like CALR mutations in myelofibrosis. Blood Cancer J. 2015;5:e360.

9. Asp J, Andreasson B, Hansson U, Wasslavik C, Abelsson J, Johansson P, et al. Mutation status of essential thrombocythemia and primary myelofibrosis defines clinical outcome. Haematologica. 2016;101:e129-32.

10. JAKAFI ${ }^{\circledR}$ (ruxolitinib). Full Prescribing Information, Incyte Corporation. Wilmington, DE, 2016. Available at: https://www.jakafi.com/pdf/prescribinginformation.pdf. Accessed 8 Aug 2016.

11. JAKAVI ${ }^{\circledast}$ (ruxolitinib). EU Summary of Product Characteristics. Basel, Switzerland: Novartis Pharmaceuticals Corporation; 2015.

12. Verstovsek S, Mesa RA, Gotlib J, Levy RS, Gupta V, DiPersio JF, et al. A double-blind, placebo-controlled trial of ruxolitinib for myelofibrosis. N Engl J Med. 2012;366:799-807.

13. Harrison C, Kiladjian JJ, Al-Ali HK, Gisslinger H, Waltzman R, Stalbovskaya V, et al. JAK inhibition with ruxolitinib versus best available therapy for myelofibrosis. N Engl J Med. 2012;366:787-98.

14. Verstovsek S, Mesa RA, Gotlib J, Levy RS, Gupta V, DiPersio JF, et al. The clinical benefit of ruxolitinib across patient subgroups: analysis of a placebocontrolled, phase III study in patients with myelofibrosis. Br J Haematol. 2013;161:508-16.

15. Verstovsek S, Mesa RA, Gotlib J, Levy RS, Gupta V, DiPersio JF, et al. Efficacy, safety, and survival with ruxolitinib in patients with myelofibrosis: results of a median 3-year follow-up of COMFORT-I. Haematologica. 2015;100:479-88.

16. Cervantes F, Vannucchi AM, Kiladjian JJ, Al-Ali HK, Sirulnik A, Stalbovskaya V, et al. Three-year efficacy, safety, and survival findings from COMFORT-II, a phase 3 study comparing ruxolitinib with best available therapy for myelofibrosis. Blood. 2013;122:4047-53.

17. Harrison CN, Vannucchi AM, Kiladjian JJ, Al-Ali HK, Gisslinger H, Knoops L, et al. Long-term findings from COMFORT-II, a phase 3 study of ruxolitinib vs best available therapy for myelofibrosis. Leukemia. 2016;30:1701-7.

18. National Cancer Institute. Common Terminology Criteria for Adverse Events v3.0 (CTCAE). Available at: http://ctep.cancer.gov/protocolDevelopment/ electronic_applications/docs/ctcaev3.pdf. Accessed 18 Oct 2016.

19. Cervantes F, Dupriez B, Pereira A, Passamonti F, Reilly JT, Morra E, et al. New prognostic scoring system for primary myelofibrosis based on a study of the International Working Group for Myelofibrosis Research and Treatment. Blood. 2009;113:2895-901.

20. Mesa RA, Verstovsek S, Gupta V, Mascarenhas JO, Atallah E, Burn T, et al. Effects of ruxolitinib treatment on metabolic and nutritional parameters in patients with myelofibrosis from COMFORT-I. Clin Lymphoma Myeloma Leuk. 2015;15:214-21. e1.

21. Deininger M, Radich J, Burn TC, Huber R, Paranagama D, Verstovsek S. The effect of long-term ruxolitinib treatment on JAK2p.V617F allele burden in patients with myelofibrosis. Blood. 2015;126:1551-4.

22. Quintás-Cardama A, Vaddi K, Liu P, Manshouri T, Li J, Scherle PA, et al. Preclinical characterization of the selective JAK1/2 inhibitor INCB018424: therapeutic implications for the treatment of myeloproliferative neoplasms. Blood. 2010;115:3109-17.

23. Quintás-Cardama A, Kantarjian H, Cortes J, Verstovsek S. Janus kinase inhibitors for the treatment of myeloproliferative neoplasias and beyond. Nat Rev Drug Discov. 2011;10:127-40.

24. Mesa RA, Cortes J. Optimizing management of ruxolitinib in patients with myelofibrosis: the need for individualized dosing. J Hematol Oncol. 2013;6:79.

25. Gupta V, Harrison CN, Hexner EO, Al-Ali HK, Foltz L, Montgomery M, et al. The impact of anemia on overall survival in patients with 
myelofibrosis treated with ruxolitinib: an exploratory analysis of the COMFORT studies, Blood (ASH Annual Meeting Abstracts). 2015.

120:abstract 1604

26. Gangat N, Caramazza D, Vaidya R, George G, Begna K, Schwager S, et al. DIPSS plus: a refined Dynamic International Prognostic Scoring System for primary myelofibrosis that incorporates prognostic information from karyotype, platelet count, and transfusion status. J Clin Oncol. 2011;29:392-7.

27. Quintas-Cardama A, Kantarjian H, Pierce S, Cortes J, Verstovsek S. Prognostic model to identify patients with myelofibrosis at the highest risk of transformation to acute myeloid leukemia. Clin Lymphoma Myeloma Leuk. 2013;13:315-8. e2.

Submit your next manuscript to BioMed Central and we will help you at every step:

- We accept pre-submission inquiries

- Our selector tool helps you to find the most relevant journal

- We provide round the clock customer support

- Convenient online submission

- Thorough peer review

- Inclusion in PubMed and all major indexing services

- Maximum visibility for your research

Submit your manuscript at www.biomedcentral.com/submit
Biomed Central 